\title{
EDITORIAL
}

\section{Preferencias de los pacientes en la toma de decisiones en cirugía}

\section{Patient preferences in decision making in surgery}

\author{
Óscar A. Guevara \\ Profesor titular, Departamento de Cirugía, Universidad Nacional de Colombia; Cirujano Hepato-Pancreato-Biliar, \\ Instituto Nacional de Cancerología, Bogotá, D.C., Colombia
}

La relación médico-paciente ha cambiado en el tiempo y este cambio, generalmente, refleja los cambios de la sociedad. Un punto crítico de esta relación es la toma de decisiones pues, por un lado, el médico tiene una posición privilegiada respecto al conocimiento y el manejo de la información, y por otro lado, el paciente es quien dispone, en su autonomía, para autorizar o no un procedimiento diagnóstico o quirúrgico, o un tratamiento.

Hace unas décadas, la decisión de la cirugía era casi exclusiva del cirujano y el paciente jugaba un papel pasivo para aceptar o, excepcionalmente, rechazar la propuesta del cirujano. Sin embargo, el ejercicio actual de la medicina y la cirugía implica tener en cuenta los deseos y las preferencias del paciente en las decisiones de atención en salud.

Casi todas las corrientes médicas de las últimas décadas han incorporado las preferencias del paciente en la toma de decisiones. Empezó por la controversial 'medicina basada en la evidencia' que propone la toma de decisiones basada en tres grandes dominios: la 'evidencia' científica, la experiencia del médico y las preferencias del paciente. Luego surgieron la 'atención en salud centrada en el paciente' y otras, como la 'medicina personalizada', en la que le dan aún mayor relevancia a las actitudes y preferencias de los pacientes en la toma de decisiones.

Paralelamente, la investigación clínica tiende, cada vez más, a incorporar los resultados centrados en el paciente (patient reported outcomes), definidos como 'cualquier reporte del estado de la condición de salud del paciente que viene directamente del paciente, sin interpretación por el médico tratante o alguien más', como complemento de los resultados clásicos como mortalidad, morbilidad o estancia hospitalaria ${ }^{\mathrm{I}}$. En Colombia, por ejemplo, se empiezan a publicar

Palabras clave: cirugía general; toma de decisiones; uso de la información científica en la toma de decisiones en salud; atención al paciente; participación del paciente; relaciones médico-paciente.

Key words: general surgery; decision making; use of scientific information for health decision making; patient care; patient participation; physician-patient relations.

Fecha de recibido: 15/10/2019 - Fecha aceptación: 16/10/2019

Correspondencia: Óscar A. Guevara, MD, MSc., Instituto Nacional de Cancerología, ESE, Calle 1 N 9-85 Bogotá, D.C., Colombia. Teléfono: (+57) (313) 282-0897Bogotá, D.C. Colombia. Correo electrónico: oaguevarac@unal.edu.co

Citar como: Guevara ÓA. Preferencias de los pacientes en la toma de decisiones en cirugía. Rev Colomb Cir. 2019;34:318-9. https://doi.org/10.30944/20117582.505

Este es un artículo de acceso abierto bajo una Licencia Creative Commons - BY-NC-ND https://creativecommons.org/licenses/by-nc-nd/4.0/deed.es 
trabajos relacionados con la ansiedad del paciente antes de la cirugía ${ }^{2}$, lo cual pone de relieve la importancia de saber qué piensa el paciente en ese periodo.

Igualmente, estas tendencias han llevado a dar una nueva interpretación a los resultados de los estudios clínicos cuando se comparan dos procedimientos pues, muchas veces, una intervención produce unos resultados mejores que otros y, la intervención comparada, muestra como mejores otros diferentes. Esto podría usarse teniendo en cuenta las expectativas del paciente al seleccionar la intervención que mejor se ajuste a sus preferencias. Por ejemplo, en la diverticulitis del colon, la colectomía conlleva menos reintervenciones y, el lavado por laparoscopia, menos colostomías. Por lo tanto, los pacientes que busquen una solución definitiva prefieren la colectomía, mientras que aquellos que quieren evitar la colostomía a toda costa podrán optar por la segunda opción ${ }^{3}$.

Esto ha llevado a la toma de decisiones compartida (shared decision-making) entre el cirujano y el paciente, como un proceso integrador de comunicación y discusión sobre la evidencia científica y los valores del paciente y su familia, lo cual mejora la toma de decisiones ${ }^{4}$.

Esta toma de decisiones compartida entre el médico y el paciente, se ha considerado un avance, tanto en el campo de la ética médica como en el de la investigación sobre servicios de salud, y consta de cuatro pasos. En el primero, el profesional informa al paciente que se va a tomar una decisión y que su opinión es importante; en el segundo, el médico explica las opciones, sus ventajas y sus desventajas; en el tercero, se discuten las preferencias del paciente y el médico lo apoya en la deliberación; cuarto, ambos discuten sobre el deseo del paciente, para tomar la decisión de común acuerdo y, también, sobre el seguimiento 5 .

No obstante, todo este proceso requiere tiempo adicional para explicar al paciente las opciones, escucharlo, discutirlas y llegar a la toma de decisiones. Infortunadamente, tanto en la consulta externa como en la visita al paciente hospitalizado, el tiempo disponible es cada vez más limitado, por lo cual la implementación de estos procesos requiere un esfuerzo en todo el sistema.

Algunos autores han denominado la 'macdonalización de la medicina' al traslado de algunos principios de los restaurantes de comida rápida a la medicina. Esto incluye: la eficiencia, a veces mal entendida, como una reducción del tiempo de contacto con el paciente; la cantidad que predomina sobre la calidad; la uniformidad en los tiempos de la consulta; el abuso de las listas de chequeo; la productividad por encima de otras consideraciones, y la estandarización excesiva del tratamiento según el tipo de enfermedad, sin tener en cuenta las necesidades individuales de los pacientes ${ }^{6}$.

En conclusión, es importante que los cirujanos nos acerquemos al paciente con explicaciones suficientes y en términos sencillos sobre su enfermedad y, especialmente, sobre las opciones terapéuticas y las alternativas, para tomar decisiones compartidas con el paciente y su familia. Esto requiere un esfuerzo del sistema de salud y de las instituciones, para proporcionar los espacios y el tiempo suficientes para facilitar estos diálogos, que lleven a una mayor satisfacción del paciente y la familia en su paso por los servicios de salud, que es el objetivo final de la atención médica.

\section{Referencias}

I. Kargo AS, Coulter A, Jensen PT, Steffensen KD. Proactive use of PROMs in ovarian cancer survivors: A systematic review. J Ovarian Res. 2019;12:63.

2. Quintero A, Yasnó DA, Riveros OL, Castillo J, Borráez BA. Ansiedad en el paciente prequirúrgico: un problema que nos afecta a todos. Rev Colomb de Cir. 2019;32:II5-20.

3. Chhabra KR, Sacks GD, Dimick JB. Surgical decision making: Challenging dogma and incorporating patient preferences. JAMA. 2017;317:357-8.

4. Boss EF, Mehta N, Nagarajan N, Links A, Berke JR, Berger Z,et al. Shared decision making and choice for elective surgical care: A systematic review. Otolaryngol Head Neck Surg. 20I6;154:405-20.

5. Stiggelbout AM, Pieterse AH, De Haes JCJM. Shared decision making: Concepts, evidence, and practice. Patient Educ Couns. 2015;98:II72-9.

6. Dorsey ER, Ritzer G. The McDonaldization of Medicine. JAMA Neurol. 20I6;73:15-6. 